\title{
Differences in Sirtuin Regulation in Response to Calorie Restriction in Cryptococcus neoformans
}

\author{
Tejas Bouklas ${ }^{1, *}$, Lindsey Masone ${ }^{1}$ and Bettina C. Fries ${ }^{2}$ \\ 1 Department of Biomedical Sciences, Long Island University-Post, Brookville, NY 11548, USA; \\ lindsey.masone@my.liu.edu \\ 2 Department of Medicine (Division of Infectious Diseases) and Department of Molecular Genetics \\ and Microbiology, Stony Brook University, Stony Brook, NY 11794 USA; \\ bettina.fries@stonybrookmedicine.edu \\ * Correspondence: tejas.bouklas@liu.edu; Tel.: +91-516-299-2695
}

Received: 26 January 2018; Accepted: 16 February 2018; Published: 18 February 2018

\begin{abstract}
Cryptococcus neoformans successfully replicates in low glucose in infected patients. In the serotype A strain, H99, growth in this condition prolongs lifespan regulated by SIR2, and can be modulated with SIR2-specific drugs. Previous studies show that lifespan modulation of a cryptococcal population affects its sensitivity to antifungals, and survival in an infection model. Sirtuins and their role in longevity are conserved among fungi; however, the effect of glucose starvation is not confirmed even in Saccharomyces cerevisiae. Lifespan analysis of C. neoformans strains in low glucose showed that $37.5 \%$ exhibited pro-longevity, and lifespan of a serotype D strain, RC2, was shortened. Transcriptome comparison of $\mathrm{H} 99$ and RC2 under calorie restriction demonstrated differences, confirmed by real-time PCR showing that SIR2, TOR1, SCH9, and PKA1 expression correlated with lifespan response to calorie restriction. As expected, $\mathrm{RC} 2$-sir $2 \Delta$ cells exhibited a shortened lifespan, which was reconstituted. However, shortened lifespan from calorie restriction was independent of SIR2. In contrast to $\mathrm{H} 99$ but consistent with altered SIR2 regulation, SIR2-specific drugs did not affect outcome of RC2 infection. These data suggest that SIR2 regulation and response to calorie restriction varies in C. neoformans, which should be considered when Sirtuins are investigated as potential therapy targets for fungal infections.
\end{abstract}

Keywords: fungus; pathogen; sirtuins; aging; resilience; glucose

\section{Introduction}

Cryptococcus neoformans is a fungal pathogen that predominantly affects immunocompromised individuals, such as HIV/AIDS patients, in whom it causes chronic meningoencephalitis (CME). Patients with this diagnosis primarily die from $\mathrm{CME}$, with the disease following either an acute infection or reactivation of a latent infection [1]. C. neoformans' ability to evade host immunity combined with its ability to replicate in the host poses a challenge to effective recovery. Despite the availability of anti-retroviral and anti-fungal therapy to circumvent these challenges, more than $80 \%$ mortality is seen in over 220,000 people affected by cryptococcosis annually [2].

C. neoformans is a haploid fungus that predominantly reproduces asexually during its course of infection [3]. Specifically, it undergoes asymmetric mitotic divisions, the sum of which determines its replicative lifespan (RLS) [4]. During these divisions, aging mother cells demonstrate phenotypic changes that include an increased cell size, which has been described in Saccharomyces cerevisiae, Candida albicans, Schizosaccharomyces pombe [5-7], and documented for C. neoformans by our laboratory [8,9]. Similar to these fungi, old C. neoformans cells cease division when RLS is completed.

Several factors have been implicated in the regulation of RLS, the most widely described and oldest intervention being calorie restriction (CR). Studies had originally shown that nutrient sensors, 
termed sirtuins [10], mediate this process; in fact, in S. cerevisiae, an extra copy of SIR2 increases RLS, whereas its loss decreases RLS [11]. However, this has since been challenged [12], primarily because longevity of yeast mother cells can be altered by affecting levels of calorie restriction (CR). Specifically, these studies have concluded that, in S. cerevisiae, Sir2 is not necessarily required for the longevity benefits gained from CR [13]. CR, achieved by growth in $0.2 \%$ or less glucose led to a significant increase in RLS, in several S. cerevisiae strains and in C. neoformans strain, H99 [11,14]. Such CR has also been implicated in affecting PKA and Sch9, which are nutrient-responsive protein kinases shown to regulate RLS in S. cerevisiae $[15,16]$. However, the majority of longevity-mediating genes identified in S. cerevisiae are involved in the Target of Rapamycin (TOR) pathway [17].

An earlier study from our laboratory was the first to characterize the contribution of SIR2 to cryptococcal aging [14]. In that study, SIR2 was chemically activated or inhibited to modulate the RLS, and subsequently the resilience of $C$. neoformans strain, H99, which is the standard laboratory strain used by most investigators. In this new study, we generated a sir $2 \Delta$ mutant (CNJ02940) in another $C$. neoformans strain, RC2, to test whether this effect was universal for C. neoformans. SIR2 expression, in addition to other genes that influence longevity under $C R$, was investigated with several low passaged clinical C. neoformans strains.

\section{Materials and Methods}

\subsection{Ethics Statement}

Vertebrate animal experiments were carried out with the approval of the Albert Einstein College of Medicine Institute for Animal Studies (protocol \#20091015, approved 29 January 2010), and adhere to any federal, state, local, and institutional guidelines.

\subsection{Strains}

C. neoformans strains used in this study are listed in Table S1. Prior to experiments, strains were maintained at $-80^{\circ} \mathrm{C}$, then sub-streaked to single colonies at least three times on standard media at $37^{\circ} \mathrm{C}$, including Yeast Extract Peptone (YEP) agar or broth (Difco) with $0.05 \%$ or $2 \%$ dextrose (Fisher), or isonicotinamide (INAM) as previously described [18]. Escherichia coli with plasmids, pJAF1, pJAF13, and pUC19 were grown on Luria Bertani (LB) agar (Fisher, Fair Lawn, NJ, USA) with ampicillin and have been described elsewhere [19].

\subsection{Growth Curves}

C. neoformans cells were grown overnight in YEP broth with $2 \%$ dextrose at $37^{\circ} \mathrm{C}$ with agitation, then diluted to an OD of 0.01 , and grown in YEP with $0.05 \%$ or $2 \%$ dextrose. Optical density was measured every $30 \mathrm{~min}$ for $48 \mathrm{~h}$ in a Bioscreen-C Automated Growth Curve Analysis System (Growth Curves USA, Piscataway, NJ, USA), and used to generate a growth curve.

\subsection{Replicative and Chronological Lifespan}

RLS was determined by adaptation of a previously published method in S. cerevisiae [20]. Briefly, C. neoformans cells $(n=20-40)$ for each strain were arrayed for micro-dissection on an agar plate supplemented with YEP and $0.05 \%$ or $2 \%$ dextrose, or and maintained at $37^{\circ} \mathrm{C}$. The first bud of each cell (virgin mother cell) was followed for each budding event by separating ensuing daughter cells every $1-2 \mathrm{~h}$ using a fiber optic needle (Cora Styles, Talent, OR, USA) affixed to the micromanipulator of an Axioscope A1 microscope (Zeiss, Thornwood, NY, USA). Plates were kept at $4{ }^{\circ} \mathrm{C}$ overnight to prevent excessive budding, and the experiment was concluded when cells had ceased division for $24 \mathrm{~h}$. RLS was quantitated by summing the buds made by each virgin cell, and reported as the median of all mothers cells examined. Chronological lifespan (CLS), or the length of time non-dividing cells survive, was determined by adaptation of a previously published method in S. cerevisiae [21]. Briefly, $10^{6}$ C. neoformans cells $/ \mathrm{ml}$ were grown in Yeast Peptone Dextrose (YPD) broth at $37^{\circ} \mathrm{C}$ with agitation until they reached stationary 
phase ( 3 days). All media was then removed by washing in sterile $\mathrm{dH}_{2} \mathrm{O}$, and cells were maintained in $\mathrm{dH}_{2} \mathrm{O}$ until $99.9 \%$ of the starting culture was dead. Viability of the culture was measured every $2 \mathrm{~d}$ by viable plate counts of appropriate dilutions on YPD agar.

\subsection{Disruption and Complementation of the SIR2 Gene}

For disruption of the SIR2 (CNJ02940) gene, primers were designed to its complete open reading frame sequence determined from JEC21 genome to replace it with a neomycin cassette (NEO) in wildtype $\mathrm{RC} 2$ cells. This was accomplished by homologous recombination using biolistic transformation in a Particle Delivery System-1000/He hepta system (Biorad, Hercules, CA, USA) to deliver a linearized DNA construct containing neomycin under ACT1 promoter control and a TRP1 terminator with an additional $1000 \mathrm{bp}$ of up- and downstream regions of the target sequence. NEO was amplified from plasmid pJAF1 using primers, Neo-F and Neo-R, and the ampicillin resistance gene was amplified from plasmid pUC19 using primers, pUC19-F and pUC19-R (see Table S2 for all primers used). All primers were designed with a Van91I restriction site for one-step directional cloning, and amplified products were restricted with Van91I and ligated using Quick ligase (New England Biolabs, Ipswich, MA, USA). Products were transformed into XL10 Gold cells (Agilent, Santa Clara, CA, USA), and transformants were selected on LB plates supplemented with ampicillin and confirmed by single digestion with Van91I. Transformants (RC-sir2 $\Delta$ ) were linearized using primers, RC2SIR2-Lfor and RC2SIR2-Rrev, and screened on YPD plates supplemented with $100 \mu \mathrm{g} / \mathrm{ml}$ G418 sulfate (neomycin), then further confirmed by PCR (Figure S1). For complementation, Wildtype SIR2 was amplified with its native promoter from the JEC21 genome using primers, RC2SIR2R-For and RC2SIR2R-Rev, containing Eco RV and Xho I restriction sites. The amplified gene was cloned into plasmid pJAF13, linearized using Apa I, and randomly inserted into sir $2 \Delta$ cells by biolistic transformation. Resulting transformants (RC2-sir2 $\Delta+S I R 2)$ were selected on YPD plates containing $100 \mu \mathrm{g} / \mathrm{ml}$ nourseothricin (Werner Bioagents, Jena, Germany), and confirmed by PCR.

\subsection{Phenotypic Characterization}

Cell and capsule sizes were measured on $C$. neoformans cells grown in respective media. Cells were suspended in India ink and imaged ( $n=100$ per group) at $1000 \times$ magnification on an Olympus AX70 microscope (Waltham, MA, USA) attached to a Qimaging Retiga 1300 digital camera (Qimaging, Surrey, BC, Canada), and sizes recorded in Adobe Photoshop CS5 (San Jose, CA, USA) for Macintosh. Capsule staining patterns were determined on C. neoformans cells stained with $\mathrm{mAb} 18 \mathrm{~B} 7$, which is specific to the glucuronoxylomannan component of the capsule, and visualized with fluorescein isothiocyanate-labeled goat anti-mouse immunoglobulin $\mathrm{G}$ as previously described [22]. Additional, phenotypic characterization, including switching frequencies, hydrogen peroxide disc diffusion assay, macrophage-mediated phagocytosis and killing, capsule induction, melanization, mating ability, and minimum inhibitory concentrations to various antifungals were determined as previously described by this lab and other groups $[14,19,23]$.

\subsection{Infection Studies}

Galleria mellonella infection was performed as previously described [24]. Briefly, a suspension of $2 \times 10^{4} \mathrm{C}$. neoformans cells was injected in the proleg of larvae $(n=20)$ (Vanderhorst Wholesale, Inc., St. Mary's, OH, USA). In addition, $2.5 \mathrm{nM}$ INAM in phosphate buffered saline was injected into a different proleg every $2 \mathrm{~d}$. Murine infection studies were performed as previously described [8]. Specifically, $10^{6}$ C. neoformans cells were injected intravenously or intratracheally into 6-8 week old female BALB/c mice $(n=10)$ (National Cancer Institute, Bethesda, MD, USA).

\subsection{Transcriptome Analysis}

RNA was collected for sequencing as previously published [14]. Differential gene expression and gene ontology enrichment (GO) was performed by the Genome Technology Access Centre at 
Washington University in St. Louis, MO, USA, and deposited on GEO (accession \#GSE74298). Real-time PCR was performed on RNA isolated from strains grown in YEP with $0.05 \%$ or $2 \%$ dextrose. Briefly, RNA was extracted with the RNeasy mini kit (Qiagen, Hilden, Germany) per manufacturer's instructions, with an initial step of mechanical disruption using a mini bead beater (Biospec, Bartlesville, OK, USA). RNA was then cleaned for DNA contamination using the MessageClean kit (GenHunter Corp., Nashville, TN, USA), and then real-time PCR was performed using the Power SYBR Green RNA-to- $\mathrm{C}_{\mathrm{T}}$ 1-Step kit (Applied Biosystems, Foster City, CA, USA) per the manufacturer's instructions, and using primers listed in Table S3. Expression levels were normalized against $\beta$-actin, and transcript levels were determined using the delta-delta CT method by comparing expression at strains grown in $0.05 \%$ to $2 \%$ dextrose.

\subsection{Statistics}

Statistical analyses, including Student's $t$-test and Log-rank test, Wilcoxon rank sum test, and Spearman's rank correlation were performed on Prism version 7 (Graphpad, La Jolla, CA, USA) or Microsoft Excel 2017 for Macintosh. Differences were considered significant if $p<0.05$. For transcriptome analysis, genes with a $p<0.05$ by a hypergeometric test, and a False Discovery Rate $q<0.25$ were termed significant.

\section{Results}

\subsection{Calorie Restriction Variably Affects the Replicative Lifespan of C. neoformans}

The effect of calorie restriction (CR) on the RLS of C. neoformans was determined in $0.05 \%$ glucose growth conditions, which corresponds to the glucose concentration encountered in human cerebrospinal fluid. As expected, CR extended the median RLS of strain $\mathrm{H} 99$ by $54 \%$ from 27.0 to 41.5 generations $(p<0.0001$ by Wilcoxon Rank Sum test) (Figure 1A), strain M8A by $317 \%$ from 9.0 to 37.5 generations $(p<0.0001)$ (Figure 1B), and strain W911A by $28 \%$ from 27.0 to 34.5 generations $(p<0.01)$ (Figure 1C). However, under $\mathrm{CR}$ growth conditions, we also found two clinical strains that exhibited a dramatically shortened lifespan; specifically, RC2 cells exhibited a 80\% shortened RLS from 67.5 to 13.5 generations $(p<0.0001)$ (Figure 1D), and I58 cells exhibited a 67\% shortened RLS from 61.5 to 20.0 generations $(p<0.0001)$ (Figure 1E). In addition, our lifespan analysis demonstrated that some strains had no change in lifespan under CR, including strain I114 (Figure 1F), I65 (Figure 1G), and M511B (Figure 1H). The change in RLS in response to CR was not dependent on whether the strain was Cryptococcus var. neoformans (serotype D, genotype VNIV) or var grubii (serotype A, genotype VNI). Doubling times varied among the strains (Table 1), and were consistently longer or unchanged in CR. However, growth times in CR media were not predictive of RLS ( $\rho$ not significant by Spearman rank correlation). In summary, $C R$ extended lifespan in only three out of eight $C$. neoformans strains, and dramatically shortened lifespan in two strains, whereas, in others, it had no effect indicating that SIR2 expression could be variable and needs to be investigated further.

Table 1. Doubling times of C. neoformans strains in rich media (RM) and calorie-restricted media (CR).

\begin{tabular}{cccc}
\hline Strain & Doubling Time in RM (h) & Doubling Time in CR (h) & $p$-Value \\
\hline H99 & $2.1 \pm 0.3$ & $2.8 \pm 0.2$ & 0.01 \\
M511B & $3.1 \pm 0.2$ & $3.6 \pm 0.3$ & 0.01 \\
M8A & $2.4 \pm 0.3$ & $2.2 \pm 0.2$ & $\mathrm{~ns}$ \\
I58 & $2.5 \pm 0.3$ & $2.9 \pm 0.4$ & $\mathrm{~ns}$ \\
I65 & $2.6 \pm 0.2$ & $3.0 \pm 0.3$ & 0.05 \\
I114 & $2.3 \pm 0.1$ & $4.0 \pm 0.2$ & 0.01 \\
RC2 & $2.4 \pm 0.2$ & $3.0 \pm 0.2$ & 0.01 \\
W911A & $3.4 \pm 0.3$ & $3.6 \pm 0.3$ & $\mathrm{~ns}$ \\
\hline
\end{tabular}



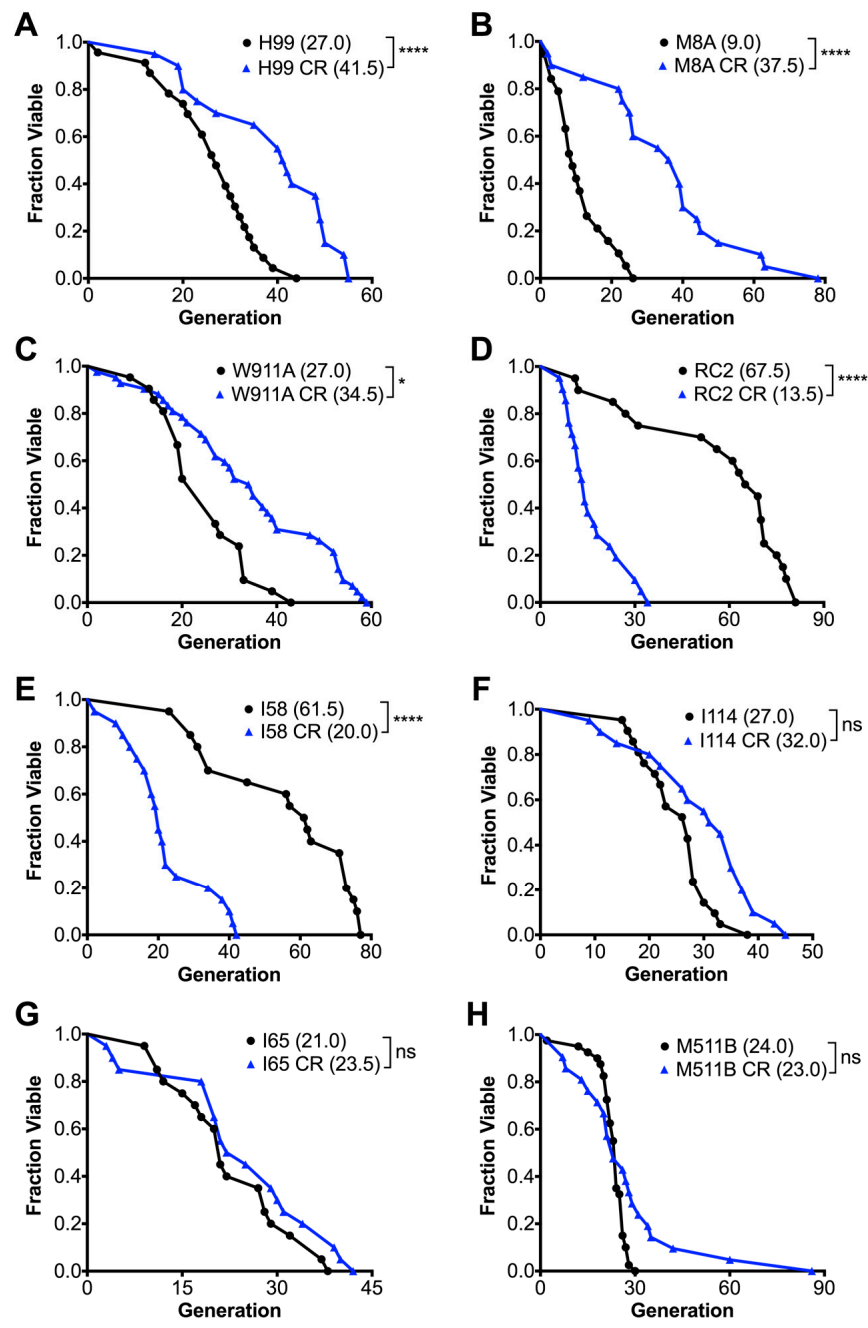

Figure 1. A variable replicative lifespan (RLS) was observed for clinical C. neoformans strains grown in calorie restricted $(\mathrm{CR})$ media. Strains grown in $\mathrm{CR}$ media were subjected to $0.05 \%$ glucose compared to $2 \%$ glucose. CR significantly extended the median RLS of (A) strain H99 by $54 \%$; (B) strain M8A by $317 \%$; and (C) strain W911A by $28 \%$. CR significantly shortened the median RLS of (D) strain RC2 by $80 \%$, and (E) strain I58 by $67 \%$. CR did not significantly affect the median RLS of strains (F) I114, (G) I65, and (H) M511B. Experiments were performed in duplicates with the respective medium ( $n=20-40$ cells). $p$-values were calculated by Wilcoxon Rank Sum test $\left({ }^{*} p<0.05,{ }^{* * * *} p<0.0001\right.$, ns not significant).

\subsection{Loss of SIR2 Has a Significant Effect on the Phenotype of Strain RC2}

Given the known role of SIR2 in replicative aging, and its CR mediated pro-longevity effect in H99 [14,23], and other yeasts [10,11], we investigated its role in CR of strain RC2, which showed such a markedly different response to CR (Figure 1D). First, SIR2 was deleted by homologous recombination in RC2 (RC2-sir2 $\Delta$, Figure S1). Additionally, the complemented strain (RC2-sir2 $\Delta+\mathrm{SIR} 2)$ was generated by non-homologous reconstitution. Loss of SIR2 in this strain, similar to what we observed in our sir $2 \Delta$ of $H 99$ [14], resulted in mildly attenuated growth in a nutrient rich medium and reduced mating ability (Table 2 and Table S4). Only minor differences in capsule size were noted in the mutant. In addition, only a mildly reduced chronological lifespan (CLS) was noted compared to the wildtype (wt). As expected, median RLS of RC2-sir $2 \Delta$ cells was shortened by $68 \%$ from 67.5 to 21.5 generations ( $p<0.0001$ by Wilcoxon Rank Sum test). This was mostly recovered ( 54.0 generations, $p<0.01$ by Wilcoxon Rank Sum test) after complementation (Figure 2A). In addition, loss of lifespan (about $67 \%$ ) of the sir $2 \Delta$ was comparable to 
that of the RC2 wt strain under CR growth conditions (Figure 2B), indicating that the CR mediated loss of lifespan in wt RC2 was not mediated or affected by SIR2 function.

Table 2. Characterization of sir2 $\Delta$ phenotypes in C. neoformans strain RC2.

\begin{tabular}{cccc}
\hline Phenotype & WT & Mutant & $p$-Value \\
\hline Doubling time in YPD & $2.0 \pm 0.2 \mathrm{~h}$ & $2.8 \pm 0.3 \mathrm{~h}$ & $<0.01$ \\
Doubling time in 0.05\% YPD & $3.0 \pm 0.2 \mathrm{~h}$ & $3.4 \pm 0.4 \mathrm{~h}$ & $\mathrm{~ns}$ \\
Mating in V8 agar & No with Kn99MATa & No with Kn99MATa & $\mathrm{ns}$ \\
Chronological lifespan & $19 \mathrm{~d}$ & $21 \mathrm{~d}$ & $\mathrm{~ns}$ \\
Phenotypic switching rate & $0.5 \times 10^{-4}$ & $0.5 \times 10^{-4}$ & $\mathrm{~ns}$ \\
Uninduced capsule size & $1.48 \pm 0.34 \mu \mathrm{m}$ & $2.19 \pm 0.28 \mu \mathrm{m}$ & $<0.01$ \\
Induced capsule size & $5.89 \pm 1.29 \mu \mathrm{m}$ & $5.65 \pm 0.92 \mu \mathrm{m}$ & $\mathrm{ns}$ \\
Total cell size & $7.25 \pm 1.09 \mu \mathrm{m}$ & $9.16 \pm 0.83 \mu \mathrm{m}$ & $<0.01$ \\
Phagocytosis index & $20.32 \%$ & $31.67 \%$ & $\mathrm{~ns}$ \\
Killing in macrophages & $59.06 \%$ & $69.36 \%$ & $\mathrm{~ns}$ \\
Colony sectoring & No sectoring & No sectoring & $\mathrm{ns}$ \\
Melanization & Same degree & Same degree & $\mathrm{ns}$ \\
GXM stain (18B7 mAb) & Same pattern & Same pattern & $\mathrm{ns}$ \\
MIC to amphotericin B & $0.125 \mu \mathrm{m} / \mathrm{mL}$ & $0.125 \mu \mathrm{g} / \mathrm{mL}$ & $\mathrm{ns}$ \\
$\mathrm{H}_{2} \mathrm{O}_{2}$ resistance & $3.1 \mathrm{~cm}$ & $3.0 \mathrm{~cm}$ & $\mathrm{~ns}$ \\
$39{ }^{\circ}$ growth & Grows & Grows & $\mathrm{ns}$ \\
\hline
\end{tabular}

GXM: Glucuronoxylomannan, MIC: Minimum Inhibitory Concentration

A

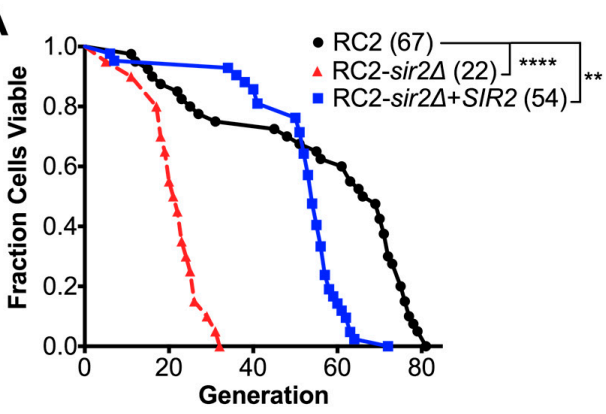

B

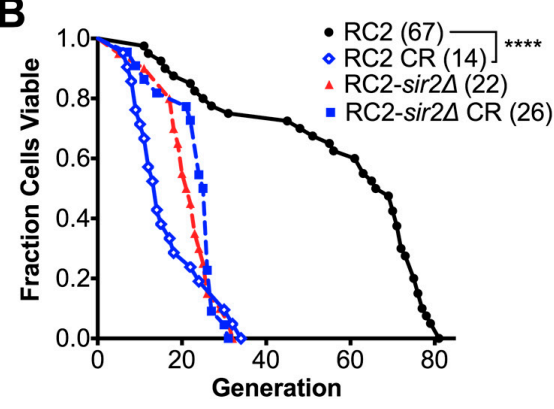

Figure 2. CR mediated loss of replicative lifespan was not mediated or affected by SIR2 in strain RC2. (A) Median RLS of RC2-sir $2 \Delta$ cells was significantly shortened by $68 \%$ compared to wt. The loss of lifespan was partially recovered after complementation; (B) under CR growth, the loss of lifespan of the sir $2 \Delta$ was comparable to the wt strain. Experiments were performed in duplicates with the respective medium ( $n=20-40$ cells). $p$-values were calculated by Wilcoxon Rank Sum test $\left({ }^{* *} p<0.01{ }^{* * * *} p<0.0001\right)$.

As expected from our studies with H99, loss of SIR2 affected some virulence. Waxmoth larvae (Galleria mellonella) infected with $2 \times 10^{4} \mathrm{RC} 2$-sir $2 \Delta$ cells demonstrated no survival difference compared to those infected with wt cells (Figure 3A). RC2-sir $2 \Delta$ cells were less virulent compared to the respective wt and complemented strains ( 97.5 vs. 38 and $11 \mathrm{~d}$ median survival, $p<0.01$ by Log Rank test) (Figure 3B) in BALB/c mice infected intratracheally with $10^{6}$ cells. A trend, albeit not significant, of lower virulence was also observed when BALB/c mice that were infected intravenously (median survival 16.5 vs. $19 \mathrm{~d}$ in RC2-sir2 $\Delta$ vs RC2-wt, respectively, ns by Log Rank test, Figure 3C). Furthermore, doubling times of wt and mutant were comparable in vitro in the low glucose growth conditions (Table 2) mimicking the brain environment. These data further supported the notion that CR did not have a life prolonging effect on RC2 and was not mediated by SIR2. 
A

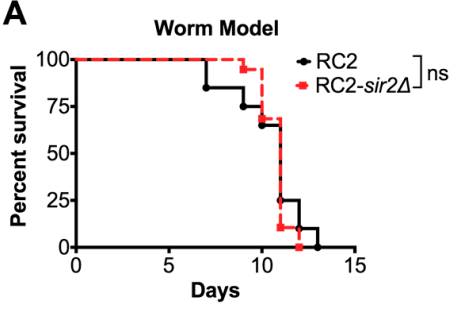

B

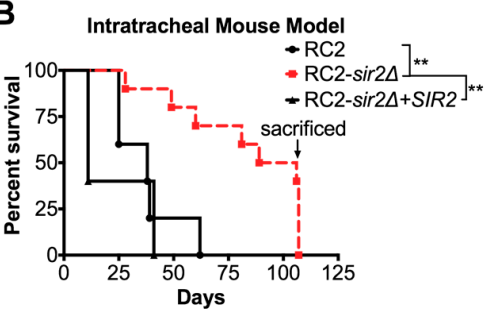

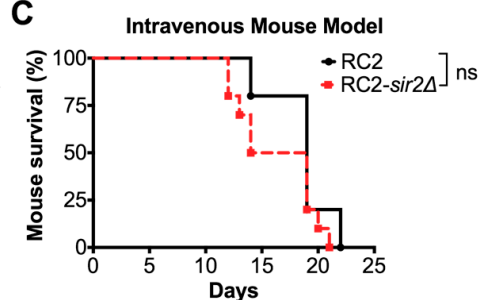

Figure 3. SIR2 loss affected virulence in some infection models. (A) Galleria mellonella larvae infected with $2 \times 10^{4} \mathrm{RC} 2$-sir $2 \Delta$ cells demonstrated no significant survival difference compared to those infected with wt cells (ns by Log Rank test); (B) BALB/c mice infected intratracheally with $10^{6}$ RC2-sir $2 \Delta$ cells survived significantly longer than those infected with the wt and complemented strains $(97.5 \mathrm{vs} .38$ and $11 \mathrm{~d}$ median, $p<0.01$ by Log Rank test); (C) BALB/c mice infected intravenously with $10^{6}$ RC2-sir $2 \Delta$ cells demonstrated no significant survival difference compared to those infected with wt cells (median survival 16.5 vs. $19 \mathrm{~d}$ in mutant vs. wt, ns by Log Rank test). Experiments were performed in duplicates ( $n=10$ mice). $p$-values were calculated by Wilcoxon Rank Sum test (** $p<0.01$, ns not significant).

\subsection{Gene Regulation of C. neoformans under Calorie Restriction}

The striking difference of CR on the RLS of cryptococcal strains, H99 and RC2, led us to compare their transcriptional response when grown in CR conditions using the available dataset published by our group (GEO accession \#GSE74298). Under CR, the two strains showed markedly different transcriptional responses. We previously reported in the $\operatorname{sir} 2 \Delta$ of the H99 strain that 340 genes/presumed genes were significantly regulated: 80 were upregulated, and 232 were downregulated under $C R$. In contrast, a significantly lower number of genes were regulated in the RC2-sir $2 \Delta$. Here, we found that 34 were upregulated (Figure 4A), and two were downregulated (Figure 4B) under CR. Interestingly, there was very little overlap between the two mutants consistent with their divergent response to CR (Table S5). Gene ontology analysis revealed that, in RC2, the most enriched genes were involved in translation in both rich and CR media regardless of the activity of SIR2; however, under CR and loss of SIR2, transcription and DNA replication was the most disrupted.

For specific genes that are associated with aging, the transcriptome analysis was confirmed by real-time PCR. Specifically, we showed that SIR2 was highly (11.4-fold) upregulated in H99 cells $(p<0.01)$, whereas it was 38-fold downregulated $(p<0.001)$ in RC2 cells (Figure 4C). SIR2 expression was also significantly up in strains M8A (20.2-fold) and W911A (5.7-fold), where CR had a pro-longevity effect on lifespan, and was significantly down in strain I58 (70.4-fold), where CR had a detrimental effect on lifespan. No significant regulation was observed in strains I114, I65, and M511B, where CR had no significant effect on lifespan. Similarly, the activity of TOR1, PKA1, SCH9 was differential among the examined strains. TOR1 expression was significantly down in strains M8A, H99, and W911A, and significantly up in strains RC2, I58, I114, and M511B (Figure 4D). SCH9 expression was significantly down in strains M8A, H99, and W911A, and significantly up in the remaining strains (Figure 4E). PKA1 expression was significantly down in strains M8A, H99, and W911A, and significantly up in strains RC2, I58, I65, and M511B (Figure 4F). In summary, comparative transcriptional analysis confirms the differential regulation of SIR2 among other genes in response to CR in C. neoformans strains. 
A

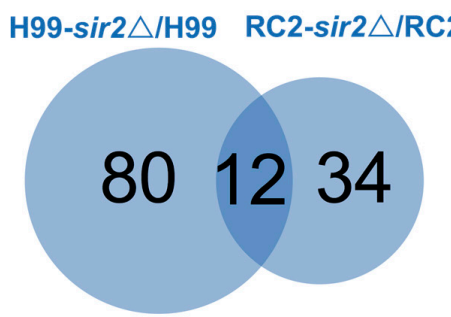

C

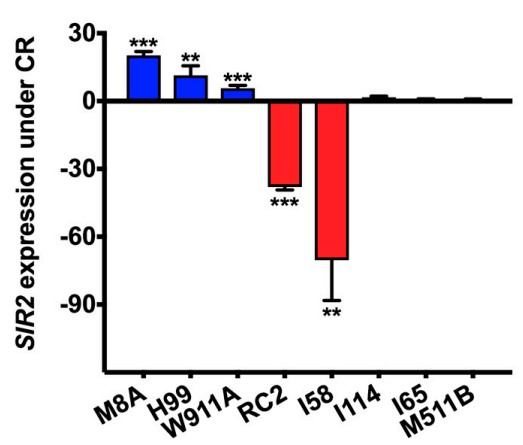

E

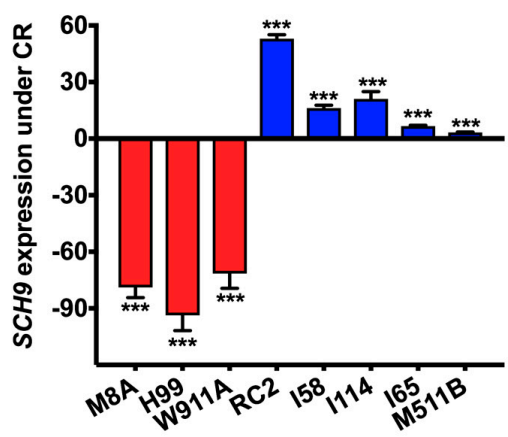

B

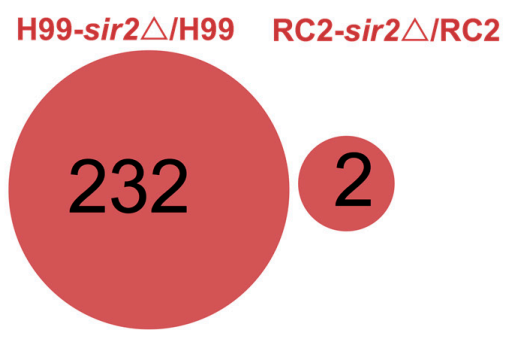

D

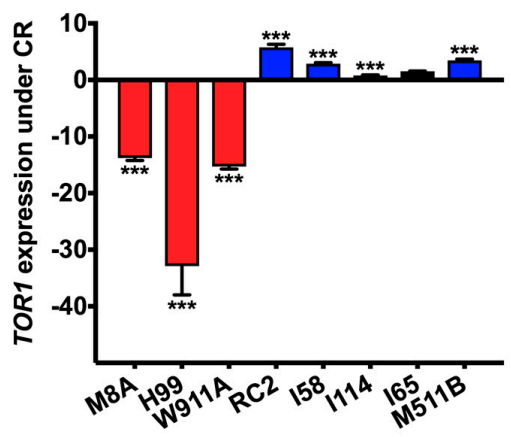

$\mathbf{F}$

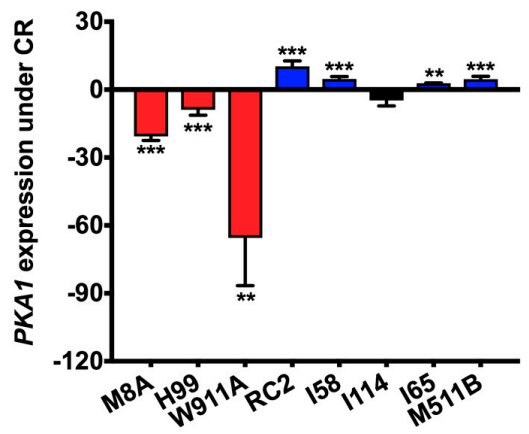

$P$-values compared to expression under RM by Student's T-test

Figure 4. Genes were differentially regulated in C. neoformans strains grown under calorie restriction. Transcriptome analysis of genes regulated under SIR2 for strains grown in CR revealed that (A) 10.5\% (12 out of 114) upregulated genes were common to strains H99 and RC2, and (B) $0 \%$ (0 out of 234) downregulated genes were common to strains H99 and RC2. RT-PCR analysis of several C. neoformans strains grown in CR revealed that (C) SIR2 expression was significantly up in strains H99, M8A, and W911A cells, and significantly down in strains RC2, and I58. No significant regulation was found in strains I114, I65, and M511B. (D) TOR1, (E) SCH9, and (F) PKA1 expression were consistently and significantly down in strains H99, M8A, and W911A cells, and consistently and significantly up in strains RC2, I58, and M511B. Some differences were found in strains I114 and I65, where expression was either significantly up or not significantly affected. For RT-PCR analysis, experiments were performed in quadruplicates and compared to strains grown in rich media. $p$-values were calculated by Student's t-test ${ }^{* *} p<0.01,{ }^{* * *} p<0.001$, ns not significant).

\subsection{SIR2 Is Differentially Regulated in RC2}

Isonicotinamide (INAM) and other SIR2 modifying drugs have been shown to extend RLS in S. cerevisiae [18], and most importantly in C. neoformans strain, H99 [14], through the action of SIR2. C. neoformans grows in the human host in the brain niche, where lower glucose concentrations are present. Hence, these drugs do not change RLS when SIR2 is mutated. Given the finding that SIR2 is differentially regulated, we tested INAM's effect on the RLS and resilience of strain RC2, where CR failed to extend RLS (Figure 1D), and the transcriptome was markedly different in the sir $2 \Delta$ and wt in response to CR (GEO accession \#GSE74298). In vitro, $25 \mathrm{mM}$ INAM had no effect of on the lifespan of sir2 $\Delta$ or 
wt cells (Figure 5A). We also investigated if the drug could alone affect resilience in the Galleria model. Repeated $2.5 \mathrm{mM}$ INAM treatment alone had no effect; larvae injected only with INAM had comparable survival to those treated with PBS as established previously [14]. As expected, INAM treatment in vivo also had no effect on survival of RC2-infected larvae (Figure 5B). Taken together, our data indicate that SIR2 regulation in C. neoformans is not consistent in all strains, and therefore SIR2 modulating drugs may also not consistently have an effect on the outcome of infection.

\section{Discussion}

This study investigates the role of SIR2 in the context of calorie restriction and its implications for the replicative lifespan of $C$. neoformans. Glucose utilization to repress aerobic respiration is a characteristic of Crabtree positive yeasts, such as S. cerevisiae and S. pombe [25]. Unlike them, C. neoformans and other pathogenic yeasts, including Candida species are Crabtree negative, and prefer respiration to fermentation regardless of glucose availability [26]. Low glucose growth conditions are present in the spinal fluid from which $C$. neoformans strains are commonly recovered. Therefore, the basidiomycete, $C$. neoformans serves as a unique model for studies in aging and CR, which are primarily done in ascomycetes. Current findings in C. neoformans are limited: a study by our laboratory [14] has shown that SIR2 modulating drugs change the replicative lifespan of a $C$. neoformans population, and by shifting its median generational age, and change its vulnerability to antifungal drugs, which are more effective on young yeast cells. Early studies in S. cerevisiae show that glucose restriction to $0.05 \%$ increases RLS by $20-40 \%[13,15,27]$, and this was attributed to SIR2 [28]. However, upon further exploration, the link between SIR2 and CR has been called into question, even in S. cerevisiae [13]. Therefore, we took a cautious approach in investigating this in C. neoformans, where SIR2 remains highly conserved [11,14].

As shown in the widely studied S. cerevisiae, several genes are also affected by CR in C. neoformans. The most prominent signature associated with aging is SIR2, which showed differential regulation in two well-studied C. neoformans strains, H99 and RC2. Both strains are highly passaged, which has been shown to result in the emergence of genotypic and phenotypic variants [29,30]. While more is known about the function of SIR2 in H99 based on a study from our group [14], not much is known in $\mathrm{RC} 2$, or low passaged clinical strains. RC2 was of particular interest because it undergoes phenotypic switching to a hypervirulent mucoid phenotype [30-32], which has a shorter RLS [8], confirming that lifespan can be regulated. The relationship between phenotypic switching and loss of RLS has also been found in Candida glabrata and is associated with poor outcome for both fungal infections $[9,33]$.

Variation in Sir2 activity stemming from strain differences has been described in S. cerevisiae [13], and we confirmed this in C. neoformans. Two laboratories have investigated different aspects of SIR2 function in H99 [14,23]. Our study investigated the effect of SIR2 modulating drugs on H99, whereas Arras et al. investigated five classes of sirtuins-SIR2, HST2, HST3, HST4, HST5-through systematic deletion in H99. Interestingly, they could not reconstitute the loss of the SIR2 function, and proposed that temporary inactivation of a sirtuin is a mechanism for C. neoformans to rapidly undergo heritable microevolutionary changes without altering its genetic material. Although both studies focused on different aspects, we found similarities and differences in several phenotypes between our $\mathrm{H} 99 \operatorname{sir} 2 \Delta$, and the mutant of the other study.

Our data now support the concept that SIR2 function varies in strains. Specifically, we found that SIR2 is important for mating, lifespan, and growth in strain RC2, which has been described in other yeasts $[10,13,18]$, and is consistent with its role in strain H99 as confirmed by our group [14]. Notably, in the study by Arras et al. [23], C. neoformans virulence associated traits were only affected by two of the five sirtuins. This is similar to our findings, where the mutant did not have a drastically altered capsule size under non-inducing conditions, and a similar response as the wt to melanization, and minimum inhibitory concentration to amphotericin B. Our data add to their findings by showing that the mutant resists stress as well as the $\mathrm{wt}$, including hydrogen peroxide stress, macrophage-mediated phagocytosis and killing, and growth at $39^{\circ} \mathrm{C}$ in a different genotype. Interestingly, no mating defect or growth difference was reported in the Arras study for $\mathrm{H} 99$ sir2 2 , whereas we found that both the RC2 
and the H99 [14] mutant grow slower in YPD; this difference may stem from the choice of method (OD measurements over 48 hours compared to overnight spot dilution), or the H99 variant. Both studies implicated SIR2 in virulence in different pulmonary murine infection models. In invertebrate models, Caenorhabditis elegans and Galleria mellonella, no survival difference was observed. The function of SIR2, and potentially compensatory sirtuins will need to be further investigated in low passaged strains, although previous data indicate that they do not regulate each other [23]. It will be of interest to investigate their function in strain $\mathrm{RC} 2$, where variants from microevolutionary pressures in the host emerge and alter the outcome of infection [30-32].

In our study, SIR2 expression was upregulated in strains that had a pro-longevity response to CR (H99, M8A, W911A), indicating its importance to longevity in C. neoformans. This was not the case for strains where CR led to loss of lifespan (RC2, I58); here, SIR2 expression was significantly down, whereas SIR2 expression was unchanged when CR had no effect on lifespan (I114, I65, M511B). For C. neoformans, SIR2 is of importance because of its role in silencing and because inherited epigenetic modifications, such as histone deacetylation, may affect the outcome of cryptococcal infection [10]. It is difficult to consolidate these findings with Arras et al. [23] since they performed a proteomic analysis on the mutants. Nonetheless, our GO analysis reveals that SIR2 has a categorical effect on carbohydrate metabolism for H99. This is now confirmed for RC2, importantly in CR growth conditions, thus providing additional insight to the role of SIR2 in this Crabtree negative yeast.

In addition, of interest was the differential transcription of TOR1, PKA, and SCH9, which in S. cerevisiae are regulated under CR and also associated with changes in RLS [28,34-36]. Deletion of TOR1 in S. cerevisiae [37,38] results in 20\% increased mean RLS; in C. neoformans, TOR1 has been suggested to be essential [39]. PKA and SCH9 have been shown to regulate RLS in S. cerevisiase $[15,16]$; specifically, a decrease in PKA activity results in an increased RLS; TOR in comparison acts upstream and parallel to PKA, whereas Sch9 acts parallel to both PKA and TOR [34,36]. Epistatic experiments involving FOB1 suggest that decreased activity of Sch9 and TOR in response to CR results in increased RLS in S. cerevisiae [13]. These studies also showed that, similar to CR, decreased TOR activity is a strain-independent mechanism to increase longevity in S. cerevisiae.

Accordingly, all three genes were significantly downregulated, in strains where $C R$ prolonged lifespan (M8A, H99, W911A). In strains where CR had a detrimental effect (RC2, I58), all were significantly upregulated. In strains where CR had no considerable influence on lifespan (I114, I65, M511B), these genes were for the most part significantly upregulated, or not significantly regulated. This upregulation likely stems from their involvement in important regulatory processes relating to metabolism, translation, and ribosome biogenesis. In S. cerevisiae, all consistently regulate the expression of ribosomal proteins [35,40], which may also be true for $C$. neoformans. In summary, comparative transcriptional analysis confirms the differential regulation of several genes implicated in $\mathrm{CR}$ in $S$. cerevisiae with $C$. neoformans. It will be of interest to study specific genes downstream of TOR that have been implicated in CR, namely Gln3 and $\operatorname{Rom} 2[37,38,41]$. Ure2, regulates the nitrogen-responsive transcription factor Gln3, and an activator of protein kinase C, Rom2. Their homologs are implicated in C. neoformans virulence, and deletion mutants exist $[42,43]$. It will also be of interest to study the genes commonly regulated under CR in the mutants of both RC2 and H99 background. Not surprisingly, since SIR2 is NAD-dependent, those involved in nicotinamide metabolism were in common: CNA07130 (dehydrogenase $\left.\mathrm{NAD}(\mathrm{P})^{+}\right), \mathrm{CNI02360}(\mathrm{NADPH}$ dehydrogenase), and CNA05810 (NAM permease). Other genes in common are implicated in C. neoformans biology; CNK02730 (sugar transporter) is responsible for intron loss and C. neoformans lineage [44]. Some genes are directly implicated in pathogenesis: CNF04430 (antiphagocytic protein) [45]; CNE02570 (succinate-fumarate antiporter), CNH02190 (malate synthase), and CNI01560 (sterol-binding protein) in early pulmonary infection [46]; the latter hints at the marked attenuated virulence in the pulmonary infection model [14,23]. Other genes include CNJ02210 (spermine transporter) in deubiquitinating [47], and oxygen sensing and sterol homeostasis [48], and CNM00600 (galactose metabolism related protein) discovered under the regulation of NRG1 in cAMP signaling [49] is implicated in capsule formation and mating. 
Our investigation into chemical modulation with isonicotinamide to stimulate SIR2 revealed differences compared to H99 [14], which highlight the variability of SIR2 function. While this pleiotropic phenomenon is disappointing from a therapeutic standpoint, it may not be entirely surprising as such variability of RLS in response to nutrients has been described in S. cerevisiae [11], where the direct role of SIR2 in response to CR has been strain-dependent. Nonetheless, our findings and the available literature on SIR2 in C. neoformans [14,23] suggest that SIR2 activity may have evolved differently in this yeast, which, opposed to Saccharomyces, has to adapt to very different host environments [26]. Further research is needed to explore the relationship of stress, generational age, and SIR2 transcription in C. neoformans strains, and how these factors alter their epigenetic landscape. It seems prudent to validate any conclusions in low passaged clinical strains, even environmental strains where virulence is attenuated, and future work will need to clarify the role of genes that may compensate for SIR 2 function in both highly passaged and low passaged strains.

Supplementary Materials: The following are available online at http:/ /www.mdpi.com/2309-608X/4/1/26/s1. Figure S1: Confirmation of the sir $2 \Delta$ mutant; Table S1: Strains used in the study; Table S2: List of primers used in the construction of the sir $2 \Delta$ mutant; Table S3: List of primers used to measure mRNA expression of select genes; Table S4: Comparison of the sir $2 \Delta$ phenotypes in the H99 and RC2 strains; Table S5: List of differentially regulated genes in RC2-sir2 $\Delta$ compared to RC2 cells grown in calorie restricted media.

Acknowledgments: Bettina C. Fries is supported by a National Institutes of Health R01 award AI127704, and Tejas Bouklas is supported by an intramural grant from the Long Island University Post Research Committee.

Author Contributions: Tejas Bouklas and Bettina C. Fries conceived and designed the experiments; Tejas Bouklas and Lindsey Masone performed the experiments; Tejas Bouklas and Bettina C. Fries analyzed the data; Tejas Bouklas and Bettina C. Fries contributed reagents/materials/analysis tools; Tejas Bouklas and Bettina C. Fries wrote the paper.

Conflicts of Interest: The authors declare no conflict of interest. The founding sponsors had no role in the design of the study; in the collection, analyses, or interpretation of data; in the writing of the manuscript, and in the decision to publish the results.

\section{References}

1. Perfect, J.R.; Casadevall, A. Cryptococcosis. Infect. Dis. Clin. North Am. 2002, 16, 837-874. [CrossRef]

2. Rajasingham, R.; Smith, R.M.; Park, B.J.; Jarvis, J.N.; Govender, N.P.; Chiller, T.M.; Denning, D.W.; Loyse, A.; Boulware, D.R. Global burden of disease of HIV-associated cryptococcal meningitis: An updated analysis. Lancet Infect. Dis. 2017, 17, 873-881. [CrossRef]

3. Alanio, A.; Desnos-Ollivier, M.; Dromer, F. Dynamics of Cryptococcus neoformans-macrophage interactions reveal that fungal background influences outcome during cryptococcal meningoencephalitis in humans. MBio 2011, 2, 41-43. [CrossRef] [PubMed]

4. Steinkraus, K.A.; Kaeberlein, M.; Kennedy, B.K. Replicative aging in yeast: The means to the end. Annu. Rev. Cell Dev. Bi. 2008, 24, 29-54. [CrossRef] [PubMed]

5. Yang, J.; Dungrawala, H.; Hua, H.; Manukyan, A.; Abraham, L.; Lane, W.; Mead, H.; Wright, J.; Schneider, B.L. Cell size and growth rate are major determinants of replicative lifespan. Cell Cycle 2011, 10, 144-155. [CrossRef] [PubMed]

6. Fu, X.H.; Meng, F.L.; Hu, Y.; Zhou, J.Q. Candida albicans, a distinctive fungal model for cellular aging study. Aging Cell 2008, 7, 746-757. [CrossRef] [PubMed]

7. Roux, A.E.; Chartrand, P.; Ferbeyre, G.; Rokeach, L.A. Fission yeast and other yeasts as emergent models to unravel cellular aging in eukaryotes. J. Gerontol. A Biol. Sci. Med. Sci. 2010, 65, 1-8. [CrossRef] [PubMed]

8. Bouklas, T.; Pechuan, X.; Goldman, D.L.; Edelman, B.; Bergman, A.; Fries, B.C. Old Cryptococcus neoformans cells contribute to virulence in chronic cryptococcosis. MBio 2013, 4, e00455-13. [CrossRef] [PubMed]

9. Jain, N.; Cook, E.; Xess, I.; Hasan, F.; Fries, D.; Fries, B.C. Isolation and characterization of senescent Cryptococcus neoformans and implications for phenotypic switching and pathogenesis in chronic cryptococcosis. Eukaryot. Cell 2009, 8, 858-866. [CrossRef] [PubMed]

10. Greiss, S.; Gartner, A. Sirtuin/Sir2 phylogeny, evolutionary considerations and structural conservation. Mol. Cells 2009, 28, 407-415. [CrossRef] [PubMed]

11. Kaeberlein, M.; McVey, M.; Guarente, L. The Sir2/3/4 complex and Sir2 alone promote longevity in saccharomyces cerevisiae by two different mechanisms. Genes Dev. 1999, 13, 2570-2580. [CrossRef] [PubMed] 
12. Guarente, L. Calorie restriction and sirtuins revisited. Genes. Dev. 2013, 27, 2072-2085. [CrossRef] [PubMed]

13. Kaeberlein, M.; Kirkland, K.T.; Fields, S.; Kennedy, B.K. Sir2-independent life span extension by calorie restriction in yeast. PLoS Biol. 2004, 2, E296. [CrossRef] [PubMed]

14. Bouklas, T.; Jain, N.; Fries, B.C. Modulation of replicative lifespan in Cryptococcus neoformans: Implications for virulence. Front Microbiol. 2017, 8, 98. [CrossRef] [PubMed]

15. Lin, S.J.; Defossez, P.A.; Guarente, L. Requirement of nad and Sir2 for life-span extension by calorie restriction in saccharomyces cerevisiae. Science 2000, 289, 2126-2128. [CrossRef] [PubMed]

16. Fabrizio, P.; Pletcher, S.D.; Minois, N.; Vaupel, J.W.; Longo, V.D. Chronological aging-independent replicative life span regulation by Msn2/Msn4 and Sod2 in saccharomyces cerevisiae. FEBS Lett. 2004, 557, 136-142. [CrossRef]

17. Schmelzle, T.; Hall, M.N. Tor, a central controller of cell growth. Cell 2000, 103, 253-262. [CrossRef]

18. McClure, J.M.; Wierman, M.B.; Maqani, N.; Smith, J.S. Isonicotinamide enhances Sir2 protein-mediated silencing and longevity in yeast by raising intracellular $\mathrm{NAD}^{+}$concentration. J. Biol. Chem. 2012, 287, 20957-20966. [CrossRef] [PubMed]

19. Jain, N.; Li, L.; Hsueh, Y.P.; Guerrero, A.; Heitman, J.; Goldman, D.L.; Fries, B.C. Loss of allergen 1 confers a hypervirulent phenotype that resembles mucoid switch variants of Cryptococcus neoformans. Infect. Immun. 2009, 77, 128-140. [CrossRef] [PubMed]

20. Park, P.U.; McVey, M.; Guarente, L. Separation of mother and daughter cells. Method. Enzymol. 2002, 351, 468-477.

21. Murakami, C.; Kaeberlein, M. Quantifying yeast chronological life span by outgrowth of aged cells. JOVE-J. Vis. Exp. 2009, 27, 3044-3054. [CrossRef] [PubMed]

22. Casadevall, A.; Cleare, W.; Feldmesser, M.; Glatman-Freedman, A.; Goldman, D.L.; Kozel, T.R.; Lendvai, N.; Mukherjee, J.; Pirofski, L.A.; Rivera, J.; et al. Characterization of a murine monoclonal antibody to Cryptococcus neoformans polysaccharide that is a candidate for human therapeutic studies. Antimicrob. Agents Ch. 1998, 42, 1437-1446.

23. Arras, S.D.M.; Chitty, J.L.; Wizrah, M.S.I.; Erpf, P.E.; Schulz, B.L.; Tanurdzic, M.; Fraser, J.A. Sirtuins in the phylum basidiomycota: A role in virulence in Cryptococcus neoformans. Sci. Rep. 2017, 7, 46567. [CrossRef] [PubMed]

24. Cotter, G.; Doyle, S.; Kavanagh, K. Development of an insect model for the in vivo pathogenicity testing of yeasts. FEMS Immunol. Med. Microbiol. 2000, 27, 163-169. [CrossRef] [PubMed]

25. Skinner, C.; Lin, S.J. Effects of calorie restriction on life span of microorganisms. Appl. Microbiol. Biotechnol. 2010, 88, 817-828. [CrossRef] [PubMed]

26. Ene, I.V.; Brunke, S.; Brown, A.J.; Hube, B. Metabolism in fungal pathogenesis. Cold Spring Harb. Perspect. Med. 2014, 4, a019695. [CrossRef] [PubMed]

27. Lin, S.J.; Kaeberlein, M.; Andalis, A.A.; Sturtz, L.A.; Defossez, P.A.; Culotta, V.C.; Fink, G.R.; Guarente, L. Calorie restriction extends saccharomyces cerevisiae lifespan by increasing respiration. Nature 2002, 418, 344-348. [CrossRef] [PubMed]

28. Kaeberlein, M.; Powers, R.W., 3rd; Steffen, K.K.; Westman, E.A.; Hu, D.; Dang, N.; Kerr, E.O.; Kirkland, K.T.; Fields, S.; Kennedy, B.K. Regulation of yeast replicative life span by TOR and SCH9 in response to nutrients. Science 2005, 310, 1193-1196. [CrossRef] [PubMed]

29. Franzot, S.P.; Mukherjee, J.; Cherniak, R.; Chen, L.C.; Hamdan, J.S.; Casadevall, A. Microevolution of a standard strain of Cryptococcus neoformans resulting in differences in virulence and other phenotypes. Infect. Immun. 1998, 66, 89-97. [PubMed]

30. Fries, B.C.; Taborda, C.P.; Serfass, E.; Casadevall, A. Phenotypic switching of Cryptococcus neoformans occurs in vivo and influences the outcome of infection. J. Clin. Invest. 2001, 108, 1639-1648. [CrossRef] [PubMed]

31. Goldman, D.L.; Fries, B.C.; Franzot, S.P.; Montella, L.; Casadevall, A. Phenotypic switching in the human pathogenic fungus Cryptococcus neoformans is associated with changes in virulence and pulmonary inflammatory response in rodents. Proc. Natl. Acad. Sci. USA 1998, 95, 14967-14972. [CrossRef] [PubMed]

32. Fries, B.C.; Goldman, D.L.; Cherniak, R.; Ju, R.; Casadevall, A. Phenotypic switching in Cryptococcus neoformans results in changes in cellular morphology and glucuronoxylomannan structure. Infect. Immun. 1999, 67, 6076-6083. [PubMed]

33. Bouklas, T.; Alonso-Crisostomo, L.; Szekely, T., Jr.; Diago-Navarro, E.; Orner, E.P.; Smith, K.; Munshi, M.A.; Del Poeta, M.; Balazsi, G.; Fries, B.C. Generational distribution of a candida glabrata population: Resilient old cells prevail, while younger cells dominate in the vulnerable host. PLoS Pathog. 2017, 13, e1006355. [CrossRef] [PubMed] 
34. Zurita-Martinez, S.A.; Cardenas, M.E. Tor and cyclic amp-protein kinase a: Two parallel pathways regulating expression of genes required for cell growth. Eukaryot. Cell 2005, 4, 63-71. [CrossRef] [PubMed]

35. Martin, D.E.; Soulard, A.; Hall, M.N. Tor regulates ribosomal protein gene expression via PKA and the forkhead transcription factor FHL1. Cell 2004, 119, 969-979. [CrossRef] [PubMed]

36. Pedruzzi, I.; Dubouloz, F.; Cameroni, E.; Wanke, V.; Roosen, J.; Winderickx, J.; De Virgilio, C. TOR and PKA signaling pathways converge on the protein kinase Rim15 to control entry into G0. Mol. Cell 2003, 12, 1607-1613. [CrossRef]

37. Helliwell, S.B.; Howald, I.; Barbet, N.; Hall, M.N. TOR2 is part of two related signaling pathways coordinating cell growth in saccharomyces cerevisiae. Genetics 1998, 148, 99-112. [PubMed]

38. Beck, T.; Hall, M.N. The tor signalling pathway controls nuclear localization of nutrient-regulated transcription factors. Nature 1999, 402, 689-692. [CrossRef] [PubMed]

39. Davidson, R.C.; Blankenship, J.R.; Kraus, P.R.; de Jesus Berrios, M.; Hull, C.M.; D'Souza, C.; Wang, P.; Heitman, J. A PCR-based strategy to generate integrative targeting alleles with large regions of homology. Microbiology 2002, 148, 2607-2615. [CrossRef] [PubMed]

40. Jorgensen, P.; Rupes, I.; Sharom, J.R.; Schneper, L.; Broach, J.R.; Tyers, M. A dynamic transcriptional network communicates growth potential to ribosome synthesis and critical cell size. Genes Dev. 2004, 18, 2491-2505. [CrossRef] [PubMed]

41. Shamji, A.F.; Kuruvilla, F.G.; Schreiber, S.L. Partitioning the transcriptional program induced by rapamycin among the effectors of the tor proteins. Curr. Biol. 2000, 10, 1574-1581. [CrossRef]

42. Fuchs, B.B.; Tang, R.J.; Mylonakis, E. The temperature-sensitive role of Cryptococcus neoformans ROM2 in cell morphogenesis. PLoS One 2007, 2, e368. [CrossRef] [PubMed]

43. Varma, A.; Wu, S.; Guo, N.; Liao, W.; Lu, G.; Li, A.; Hu, Y.; Bulmer, G.; Kwon-Chung, K.J. Identification of a novel gene, URE2, that functionally complements a urease-negative clinical strain of Cryptococcus neoformans. Microbiology 2006, 152, 3723-3731. [CrossRef] [PubMed]

44. Stajich, J.E.; Dietrich, F.S. Evidence of mRNA-mediated intron loss in the human-pathogenic fungus Cryptococcus neoformans. Eukaryot. Cell 2006, 5, 789-793. [CrossRef] [PubMed]

45. Williams, V.; Del Poeta, M. Role of glucose in the expression of Cryptococcus neoformans antiphagocytic protein 1, app1. Eukaryot. Cell 2011, 10, 293-301. [CrossRef] [PubMed]

46. Hu, G.; Cheng, P.Y.; Sham, A.; Perfect, J.R.; Kronstad, J.W. Metabolic adaptation in Cryptococcus neoformans during early murine pulmonary infection. Mol. Microbiol. 2008, 69, 1456-1475. [CrossRef] [PubMed]

47. Fang, W.; Price, M.S.; Toffaletti, D.L.; Tenor, J.; Betancourt-Quiroz, M.; Price, J.L.; Pan, W.H.; Liao, W.Q.; Perfect, J.R. Pleiotropic effects of deubiquitinating enzyme ubp5 on growth and pathogenesis of Cryptococcus neoformans. PLoS One 2012, 7, e38326. [CrossRef] [PubMed]

48. Chang, Y.C.; Bien, C.M.; Lee, H.; Espenshade, P.J.; Kwon-Chung, K.J. Sre1p, a regulator of oxygen sensing and sterol homeostasis, is required for virulence in Cryptococcus neoformans. Mol. Microbiol. 2007, 64, 614-629. [CrossRef] [PubMed]

49. Cramer, K.L.; Gerrald, Q.D.; Nichols, C.B.; Price, M.S.; Alspaugh, J.A. Transcription factor nrg1 mediates capsule formation, stress response, and pathogenesis in Cryptococcus neoformans. Eukaryot. Cell 2006, 5, 1147-1156. [CrossRef] [PubMed]

(C) 2018 by the authors. Licensee MDPI, Basel, Switzerland. This article is an open access article distributed under the terms and conditions of the Creative Commons Attribution (CC BY) license (http://creativecommons.org/licenses/by/4.0/). 